\title{
Effects of speaking rate on mora timing organization in producing Japanese contrastive geminate/single consonants and long/short vowels by native and Chinese speakers
}

\author{
Haiping Jia ${ }^{1}$, Hiroki Mori ${ }^{2}$ and Hideki Kasuya ${ }^{2, *}$ \\ ${ }^{1}$ Graduate School of Engineering, Utsunomiya University, \\ 7-1-2, Yoto, Utsunomiya, 321-8585 Japan \\ ${ }^{2}$ Faculty of Engineering, Utsunomiya University, \\ 7-1-2, Yoto, Utsunomiya, 321-8585 Japan
}

(Received 3 June 2005, Accepted for publication 30 June 2005 )

Keywords: Mora timing organization, Speaking rate, Regression analysis, L2 speech PACS number: 43.70.Kv [DOI: $10.1250 /$ ast.26.533]

\section{Introduction}

It is widely accepted that the prosodic structure of Japanese can be reasonably analyzed by introducing 'mora' as a phonological unit, which consists of the CV-type syllable, first part of a geminate consonant, second part of a long vowel and moraic nasal [1]. Phonetic realization of the mora has been continually studied primarily in terms of the segment duration involved in the production [2-6], and perception of the mora $[7,8]$.

The mora timing organization for producing contrastive geminate/single consonants and long/short vowels is a difficult task for non-native speakers of Japanese and has been investigated from pedagogical viewpoints [9-11]. Non-native speakers of Japanese often reveal incorrect timing organization in the production of a phrase including a geminate consonant or a long vowel at various speaking rates, even though they maneuver the timing organization skillfully at a regular speaking rate [12].

This paper investigates characteristics of the segment durations involved in the production of contrastive geminate/ single consonants and long/short vowels by native Japanese speakers (JSb) and Chinese-speaking subjects of Japanese $(\mathrm{CSb})$, when the speaking rate is changed. Although Yi reported the effects of speaking rate on the vowel length distinction for native and non-native speakers of Japanese [13] and Hirata for native Japanese speakers [14], this paper attempts to clarify the durational structure of the special morae in Japanese (first part of the geminate consonants and second part of the long vowels) in more specific ways and to compare the mora timing organization of Chinese-speaking subjects of Japanese with that of native Japanese subjects.

\section{Speech materials}

A set of words including geminate consonants (C1C2) and long vowels (V1V2) was prepared:

/goseku/(Gose-ward)-/gosekku/(five seasonal festivals)/goseeku/(polite form of "idiomatic phrase"),

where $\mathrm{C} 1$ indicates the first part of the geminate stop consonant $/ \mathrm{kk} /$ and V2 the second part of the long vowel /ee/.

\footnotetext{
*e-mail: kasuya@klab.jp
}

Six native Japanese subjects (JS-A, JS-B, JS-C, JS-D, JS-E, and JS-F) and five Chinese-speaking subjects (CS-A, CS-B, CS-C, CS-D, and CS-E) read the word set five times and seven times, respectively, in this order at four speaking rates: regular, slow, fast, and very fast. Each word (***) was placed in a carrier sentence, "Kore o $* * *$ to iimasuka?" (Do you call this $* * * ?)$. All the subjects except for JS-D, JS-E and CS-E were males.

Examples of the speech waveform of JS-F at a regular speaking rate are illustrated in Fig. 1, where sound segment boundaries are marked in by a vertical line for the beginning of [s], the end of [e], and the beginning of the explosion of [k]. The boundaries were located manually by looking carefully at the sound spectrograms and waveforms; $T_{\mathrm{S}}, T_{\mathrm{e}}$ and $T_{\mathrm{X}}$ were marked for /goseku/, $T_{\mathrm{CS}}$ and $T_{\mathrm{CX}}$ for /gosekku/, and $T_{\mathrm{VS}}$ and $T_{\mathrm{Ve}}$ for /goseeku/ as shown in the figure. The segment durations of $\mathrm{C} 1, D_{\mathrm{C} 1}$ and $\mathrm{V} 2, D_{\mathrm{V} 2}$ were respectively determined in milliseconds as:

$$
\begin{aligned}
& D_{\mathrm{C} 1}=\left(T_{\mathrm{CX}}-T_{\mathrm{CS}}\right)-\left(T_{\mathrm{X}}-T_{\mathrm{S}}\right) \\
& D_{\mathrm{V} 2}=\left(T_{\mathrm{Ve}}-T_{\mathrm{VS}}\right)-\left(T_{\mathrm{e}}-T_{\mathrm{S}}\right)
\end{aligned}
$$

In the measurement of $D_{\mathrm{C} 1}$ and $D_{\mathrm{V} 2}$, the syllabic unit of each word was taken into account, i.e. /se/, /sek/ and /see/. In order to compare the characteristics of the segment durations $D_{\mathrm{C} 1}$ and $D_{\mathrm{V} 2}$ of CSbs with those of JSbs, all the seven utterances of /goseku/ by the five CSbs at the four speaking rates were perceptually evaluated by a native Japanese language instructor with regard to whether the mora timing organization of their utterances was correct or not. This was because the measurement of the segment durations $D_{\mathrm{C} 1}$ and $D_{\mathrm{V} 2}$ was based on a correct utterance of /goseku/ as shown in the equations above. Once the mora timing organization of an utterance /goseku/ by a CSb at a certain speaking rate was judged incorrect, the following utterances of /gosekku/ and /goseeku/ in a corresponding set were excluded from further statistical analyses. Numbers of excluded speech samples are indicated in Table 1 for each of the subjects at the four speaking rates. Incorrect pronunciations took place mostly at slow and fast speaking rates across the speakers. In the slow utterance by CS-D, all the five incorrect pronunciations were a substitution of the single consonant $/ \mathrm{k} /$ to the geminate 


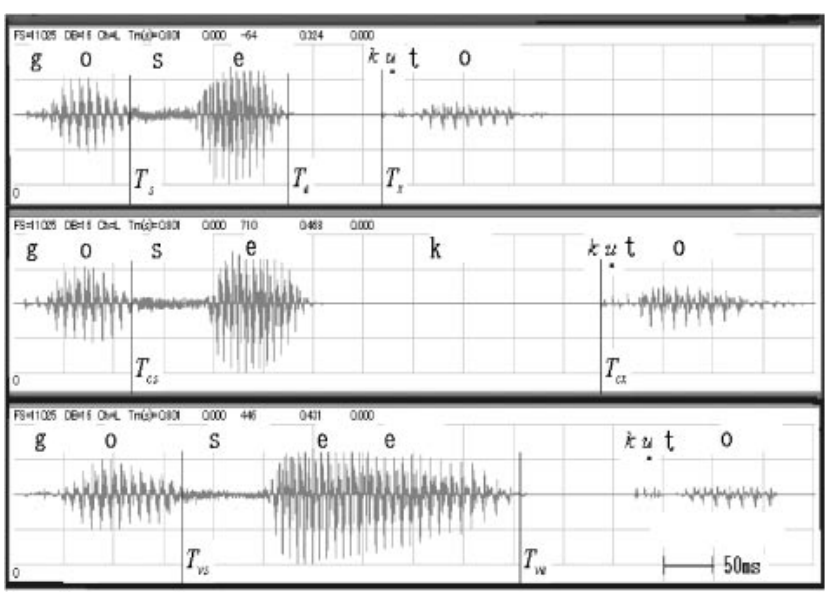

Fig. 1 An example of a set of speech waveforms /goseku/, /gosekku/ and /goseeku/, followed by the particle /to/, together with segment boundaries for the measurement of the durations.

Table 1 Number of the word set judged as incorrect utterance.

\begin{tabular}{|c|c|c|c|c|c|}
\hline \multirow{2}{*}{ Subject } & \multicolumn{4}{|c|}{ Speaking rate } & \multirow{2}{*}{ Tota } \\
\hline & Regular & Slow & Fast & Very fast & \\
\hline CS-A & 0 & 1 & 1 & 0 & 2 \\
\hline CS-B & 0 & 0 & 0 & 0 & 0 \\
\hline CS-C & 0 & 2 & 0 & 0 & 2 \\
\hline CS-D & 0 & 5 & 1 & 0 & 6 \\
\hline CS-E & 0 & 1 & 0 & 0 & 1 \\
\hline
\end{tabular}

consonant $/ \mathrm{kk} /$. The timing organization of all the utterances of Japanese subjects was judged correct.

\section{Evaluation of mora timing organization of Chinese- speaking subjects}

Three out of the seven sets of utterances of each of the CSbs were randomly selected and subjected to the evaluation of the degree of achievement of the mora timing organization. Four Japanese language instructors listened to the three sets of utterances of the five Chinese subjects and evaluated the degree on a scale of four: beginner (1), intermediate (2), advanced (3), and excellent (4), in which the excellent was given when the ability of mora timing control of a CSb was judged identical to that of native speakers of Japanese. Average scores obtained in the evaluation are shown in Table 2, together with the mean value of the average scores of each CSb. The CSbs achieved better scores for utterances of the long vowel (V2) than for those of the geminate consonant (C1), implying that the timing organization of a geminate consonant is more difficult than that of a long vowel for CSbs.

\section{Regression analysis of the durations}

Figures 2(a) and (b) illustrate respectively variations of the measured $D_{\mathrm{C} 1}$ and $D_{\mathrm{V} 2}$ against the change of speaking rate for the native Japanese speaker JSb-B. The abscissa is the average mora duration of the word. Therefore, the faster the
Table 2 Average scores of the five Chinese-speaking subjects in the achievement of the mora timing organization of $\mathrm{C} 1$ and $\mathrm{V} 2$.

\begin{tabular}{cccccc}
\hline Subject & CS-A & CS-B & CS-C & CS-D & CS-E \\
\hline $\begin{array}{c}\text { Average score } \\
\text { (C1) }\end{array}$ & 1.5 & 3.8 & 2.3 & 2.3 & 2.5 \\
$\begin{array}{c}\text { Average score } \\
\text { (V2) }\end{array}$ & 2.8 & 3.8 & 3.0 & 2.8 & 3.5 \\
$\quad$ Mean & 2.2 & 3.8 & 2.7 & 2.6 & 3.0 \\
\hline
\end{tabular}

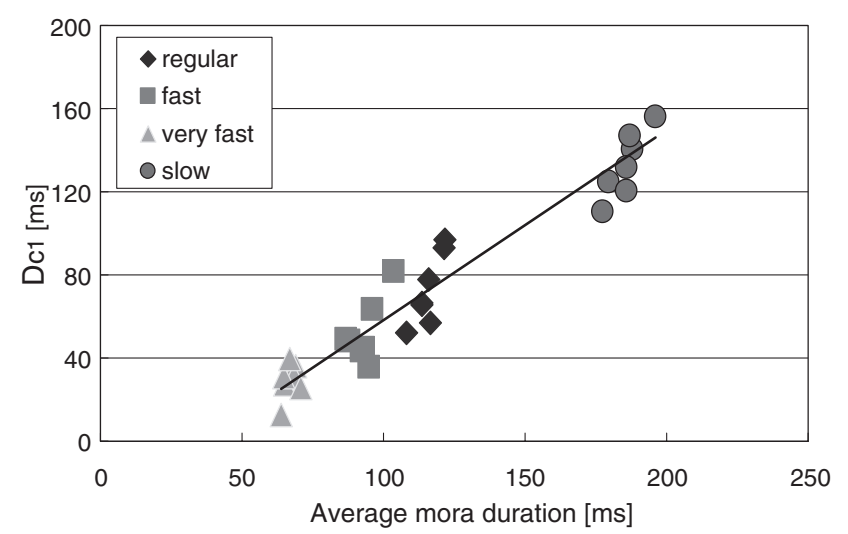

(a)

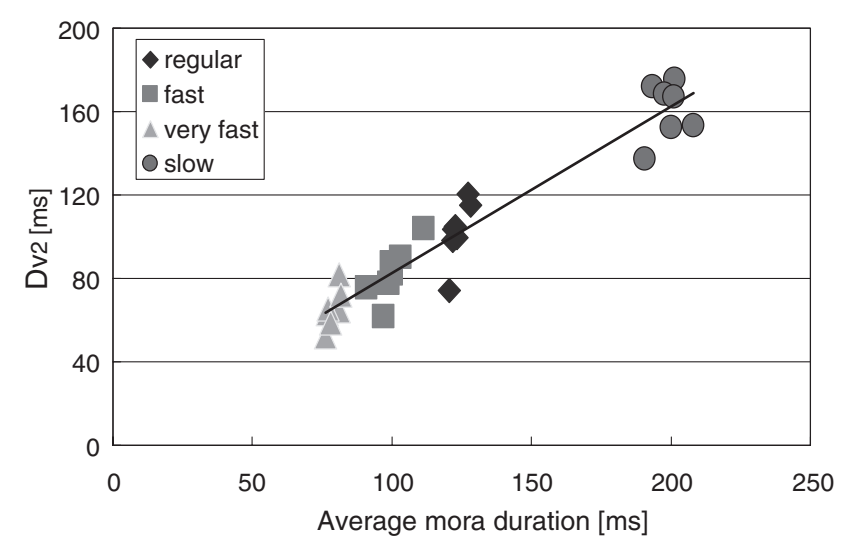

(b)

Fig. 2 (a) Relationship between measured $D_{\mathrm{C} 1}$ and average mora duration for JS-B. (b) Relationship between measured $D_{\mathrm{V} 2}$ and average mora duration for JS-B.

speaking rate, the shorter the average mora duration of the word becomes. A regression line is also depicted in the figures. It is evident from the figures that $D_{\mathrm{C} 1}$ and $D_{\mathrm{V} 2}$ lie fairly close on the straight line. The same statistical tendencies were observed in the other JSbs. Examples of $D_{\mathrm{C} 1}$ and $D_{\mathrm{V} 2}$ of CS-A, who was given the worst mean score in Table 2, and CS-B, who gained the best, are respectively shown in Figs. 3 and 4. The distribution of CS-B is very close to that of JSb-A in Fig. 1 in the sense that both yield a compact distribution along the regression line, whereas this is not the case in CS-A in Fig. 4.

The regression model is given by 


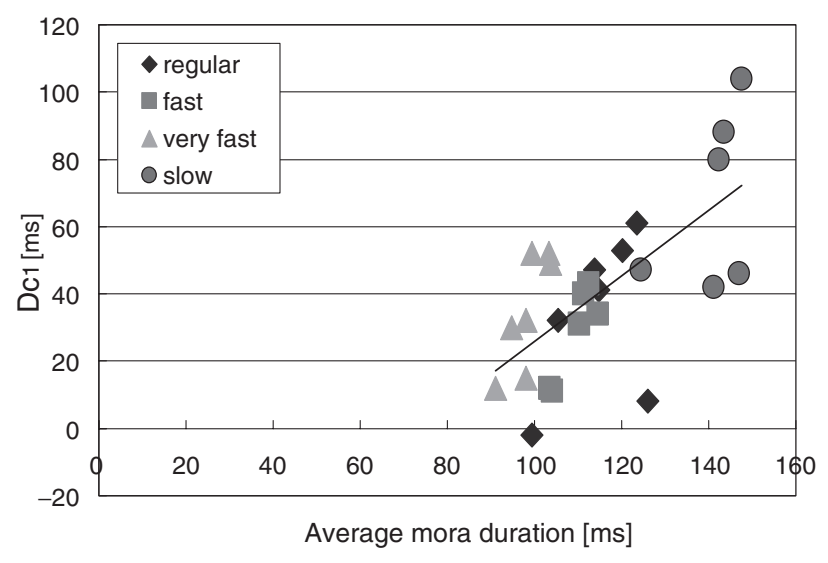

(a)

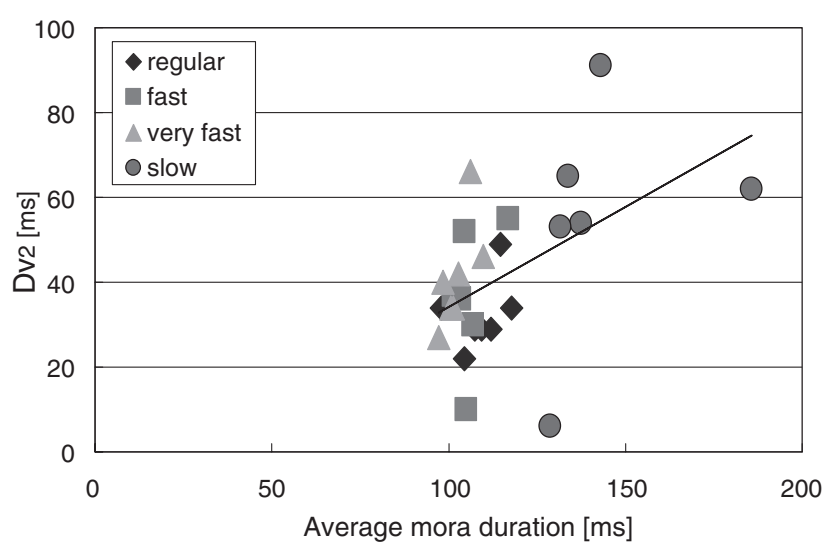

(b)

Fig. 3 (a) Relationship between measured $D_{\mathrm{C} 1}$ and average mora duration for CS-A. (b) Relationship between measured $D_{\mathrm{V} 2}$ and average mora duration for CS-A.

$$
D_{i}=a_{1 i} D_{\mathrm{a}}+a_{0 i}+\varepsilon_{i}, \quad i=\mathrm{C} 1 \text { or } \mathrm{V} 2
$$

where $D_{\mathrm{a}}$ is an average mora duration of a word “***," $a_{1 i}$ and $a_{0 i}$ are regression coefficients, and is $\varepsilon_{i}$ an error term. Statistics resulting from the regression analysis are tabulated in Tables 3 and 4 for $D_{\mathrm{C} 1}$ and $D_{\mathrm{V} 2}$, respectively. In the tables the normalized standard error (NSE) is the standard error (SE) that is normalized by a predicted value of the duration at each of the average mora durations. $\mathrm{CD}$ is the coefficient of determination and SD is the standard deviation of the average mora durations.

\section{Discussion}

The regression coefficient $a_{1}$ (slope of the line) differ little among JSbs in both the geminate consonant and the long vowel, while the coefficient $a_{0}$ (section) varies to some extent, suggesting that the speaker individuality of JSbs in the timing organization of the special morae is primarily governed by the section parameter $a_{0}$. The coefficient of determination (CD) exceeds 0.85 in all the six JSbs. These statistics evidently indicate that native Japanese speakers retain their ability to produce the same, stable mora timing organization against changes in the speaking rate and that there is a clear linear

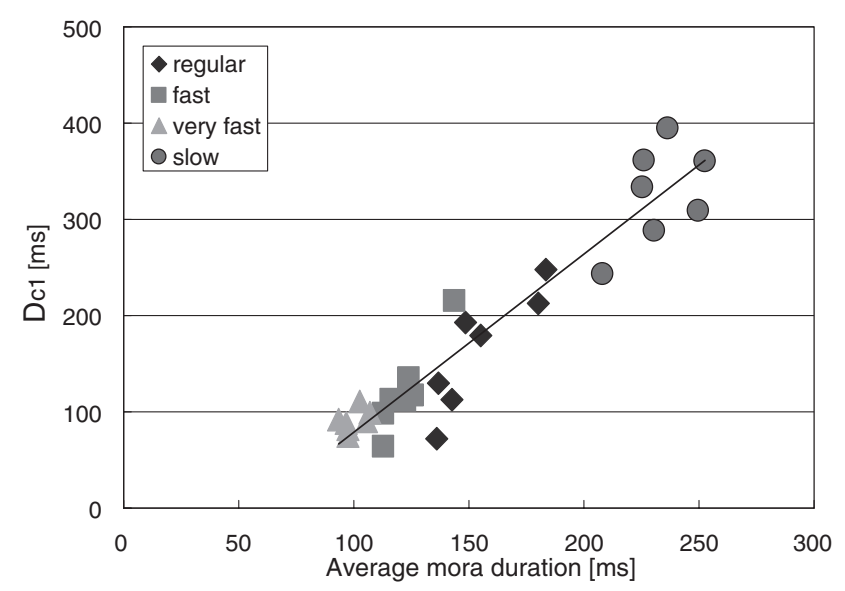

(a)

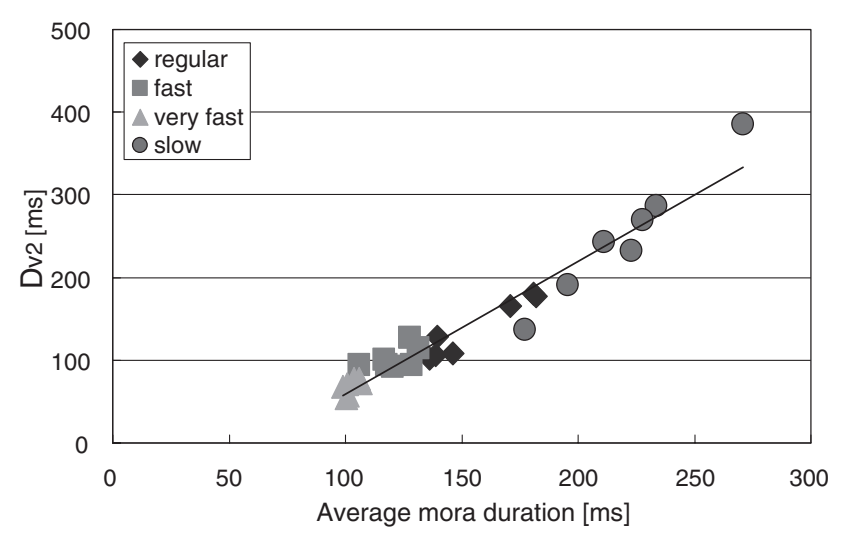

(b)

Fig. 4 (a) Relationship between measured $D_{\mathrm{C} 1}$ and average mora duration for CS-B. (b) Relationship between measured $D_{\mathrm{V} 2}$ and average mora duration for CS-B.

relationship between the duration of the $\mathrm{C} 1$ and V2 mora and the average mora duration of a word in which $\mathrm{C} 1$ and $\mathrm{V} 2$ are respectively included.

In the CSbs, on the other hand, the regression coefficients differ largely from subject to subject, indicating the existence of large individualities among the CSbs. Since the coefficient values of CS-B and CS-E are considerably different in V2 regardless of the similar high evaluation scores that they gained, they are considered to have achieved different strategies to control the mora timing of a long vowel when the speaking rate varies. In Tables 3 and 4, all the CSbs except for CS-B seem to have difficulty in controlling the speaking rate in a wide range, since the standard deviations of the average mora durations (SD) in the tables are small in most of the cases. Note that CS-B has succeeded in controlling the rate widely by employing a slightly different strategy from that of JSbs, since the $a_{1}$ and $a_{0}$ values of CS-B are considerably larger than JSbs as can be seen in the tables. CS-B has introduced much longer durations of $\mathrm{C} 1$ and $\mathrm{V} 2$ at a slow speaking rate.

By comparing Table 2 with Tables 3 and 4, the normalized standard errors (NSE) seem to show relatively good relations with the degree of achievement of the mora timing 
Table 3 Statistics obtained by regression analysis of $D_{\mathrm{C} 1}$.

\begin{tabular}{ccrrccc}
\hline Subject & $a_{1 \mathrm{C} 1}$ & \multicolumn{1}{c}{$a_{0 \mathrm{C} 1}$} & \multicolumn{1}{c}{ SE } & NSE & CD & SD \\
\hline JS-A & 0.85 & -26.5 & 20.1 & 0.28 & 0.89 & 61.3 \\
JS-B & 0.91 & -33.0 & 13.0 & 0.25 & 0.91 & 49.8 \\
JS-C & 0.99 & -39.4 & 6.1 & 0.18 & 0.96 & 57.6 \\
JS-D & 0.95 & -12.0 & 11.4 & 0.11 & 0.95 & 61.2 \\
JS-E & 0.96 & -12.2 & 9.6 & 0.11 & 0.95 & 54.1 \\
JS-F & 1.13 & -3.6 & 16.5 & 0.12 & 0.86 & 44.1 \\
CS-A & 0.97 & -71.4 & 18.6 & 0.49 & 0.46 & 22.1 \\
CS-B & 1.85 & -106.6 & 32.0 & 0.20 & 0.91 & 67.2 \\
CS-C & 1.05 & -41.0 & 18.9 & 0.44 & 0.57 & 27.1 \\
CS-D & 2.02 & -124.3 & 22.9 & 0.34 & 0.85 & 36.8 \\
CS-E & 1.52 & -106.6 & 37.2 & 0.48 & 0.52 & 31.9 \\
\hline
\end{tabular}

Table 4 Statistics obtained by regression analysis of $D_{\mathrm{V} 2}$.

\begin{tabular}{ccrrccc}
\hline Subject & $a_{1 \mathrm{~V} 2}$ & \multicolumn{1}{c}{$a_{0 \mathrm{~V} 2}$} & \multicolumn{1}{c}{ SE } & NSE & CD & SD \\
\hline JS-A & 0.83 & -5.6 & 18.6 & 0.15 & 0.90 & 51.3 \\
JS-B & 0.93 & -17.1 & 10.9 & 0.11 & 0.92 & 47.2 \\
JS-C & 0.87 & -19.8 & 14.3 & 0.16 & 0.95 & 58.2 \\
JS-D & 0.90 & -8.8 & 16.8 & 0.13 & 0.86 & 60.0 \\
JS-E & 0.95 & -21.7 & 9.1 & 0.09 & 0.96 & 55.8 \\
JS-F & 1.07 & -13.1 & 10.4 & 0.10 & 0.92 & 42.1 \\
CS-A & 0.47 & -13.1 & 16.2 & 0.36 & 0.25 & 24.6 \\
CS-B & 1.60 & -100.8 & 19.2 & 0.14 & 0.95 & 61.9 \\
CS-C & 1.53 & -72.5 & 15.9 & 0.33 & 0.78 & 28.4 \\
CS-D & 1.90 & -110.6 & 15.5 & 0.18 & 0.90 & 30.7 \\
CS-E & 0.71 & -10.1 & 18.1 & 0.23 & 0.42 & 26.4 \\
\hline
\end{tabular}

organization for $\mathrm{C} 1$; the better the score of the degree, the smaller the NSE, with an exception of CS-E. How-ever, this is not the case with V2. One of the reasons for this inconsistency is that all the CSbs are close to the advanced or excellent level of the achievement. More CSbs at beginner and intermediate levels are needed to cover a wide range of subjects.

\section{Conclusion}

It has been shown that there exists a simple structure of the mora timing organization with little individualities pertinent to a geminate consonant and a long vowel in
Japanese in case of native Japanese speakers, when the speaking rate is changed. Chinese-speaking subjects of Japanese, on the other hand, have shown large individualities of the mora timing organization, some of whom seem to use different strategies to control the mora timing from those used by native speakers and have difficulty in controlling the speaking rate in a wide range.

\section{References}

[1] H. Kubozono, Phonetics and Phonology (Kuroshio Publishers, Tokyo, 1998), pp. 47-74.

[2] M. S. Han, "The feature of duration in Japanese," Stud. Sounds, 10, 65-80 (1962).

[3] Y. Homma, "Durational relationship between Japanese stops and vowels," J. Phonet., 9, 273-281 (1981).

[4] R. F. Port, S. Al-Ani and S. Maeda, "Temporal compensation and universal phonetics," Phonetica, 37, 235-252 (1980).

[5] M. Beckman, "Segment duration and the 'mora' in Japanese," Phonetica, 39, 113-135 (1982).

[6] Y. Sagisaka and Y. Tohkura, "Phoneme duration control for speech synthesis by rule," Trans. Inst. Electr. Commun. Eng. Jpn., J67-A, 629-636 (1984).

[7] H. Fujisaki, K. Nakamura and T. Imoto, "Auditory perception of duration of speech and non-speech stimuli," in Auditory Analysis and Perception of Speech, G. Fant and M. A. Tatham, Eds. (Academic Press, London, 1975), pp. 45-64.

[8] S. Fukui, "Perception for [sic] the Japanese stop consonants with reduced and extended durations," Bull. Phonet. Soc. Jpn., 159, 9-12 (1978).

[9] M. S. Han, "The timing control of geminate and single stop consonants in Japanese: A challenge for nonnative speakers," Phonetica, 49, 102-127 (1992).

[10] T. Toda, "Interlanguage phonology: Acquisition of timing control in Japanese," Aust. Rev. Appl. Linguist., 17, 51-76 (1994).

[11] Y. Hirata, "Training native English speakers to perceive Japanese length contrasts in word versus sentence contexts," J. Acoust. Soc. Am., 116, 2384-2394 (2004).

[12] H. Jia, H. H. Mori and H. Kasuya, "Quantitative evaluation of naturalness of mora timing for Chinese speakers," Proc. Autumn Meet. Acoust. Soc. Jpn., Vol. 1, 437-438 (2004).

[13] K.-S. Yi, "The effect of articulation rate on Japanese double vowel production in Korean learners of Japanese," Tech. Rep. Inst. Electron. Inf. Commun. Eng. Jpn., TL2003-22, pp. 37-42 (2003).

[14] Y. Hirata, "Effects of speaking rate on the vowel length distinction in Japanese," J. Phonet., 32, 565-589 (2004). 